\title{
RESEARCH
}

Open Access

\section{Independent and combined effects of physical activity and body mass index on the development of Type 2 Diabetes - a meta-analysis of 9 prospective cohort studies}

Laura Cloostermans', Wanda Wendel-Vos ${ }^{1 *}$, Gerda Doornbos', Bethany Howard², Cora Lynn Craig ${ }^{3}$, Mika Kivimäki ${ }^{4}$, Adam G. Tabak ${ }^{4}$ Barbara J. Jefferis ${ }^{5}$, Kimmo Ronkainen ${ }^{6}$, Wendy J. Brown ${ }^{7}$, Susan H. S. J. Picavet ${ }^{1}$, Yoav Ben-Shlomo ${ }^{8}$, Jari Antero Laukkanen ${ }^{6,9}$, Jussi Kauhanen ${ }^{6}$ and Wanda J. E. Bemelmans ${ }^{1}$

\begin{abstract}
Background: The aim of this harmonized meta-analysis was to examine the independent and combined effects of physical activity and BMI on the incidence of type 2 diabetes.

Methods: Our systematic literature review in 2011 identified 127 potentially relevant prospective studies of which 9 fulfilled the inclusion criteria (total $N=117,878,56.2 \%$ female, mean age $=50.0$ years, range $=25-65$ years). Measures of baseline physical activity (low, intermediate, high), BMl-category [BMI < 18.4 (underweight), 18.5-24.9 (normal weight), 25.0-29.9 (overweight), 30+ (obese)] and incident type 2 diabetes were harmonized across studies. The associations between physical activity, BMI and incident type 2 diabetes were analyzed using Cox regression with a standardized analysis protocol including adjustments for age, gender, educational level, and smoking. Hazard ratios from individual studies were combined in a random-effects meta-analysis.
\end{abstract}

Results: Mean follow-up time was 9.1 years. A total of 11,237 incident type 2 diabetes cases were recorded. In mutually adjusted models, being overweight or obese (compared with normal weight) and having low physical activity (compared with high physical activity) were associated with an increased risk of incident type 2 diabetes (hazard ratios 2.33, $95 \%$ Cl 1.95-2.78; 6.10, $95 \%$ Cl: 4.63-8.04, and 1.23, $95 \%$ Cl: 1.09-1.39, respectively). Individuals who were both obese and had low physical activity had 7.4-fold (95\% Cl 3.47-15.89) increased risk of type 2 diabetes compared with normal weight, high physically active participants.

Conclusions: This harmonized meta-analysis shows the importance of maintaining a healthy weight and being physically active in diabetes prevention.

Keywords: Physical activity, Body mass index, Overweight, Obesity, Meta-analysis

\footnotetext{
* Correspondence: Wanda.Vos@rivm.nl

${ }^{1}$ National Institute for Public Health and the Environment, Bilthoven, The Netherlands

Full list of author information is available at the end of the article
} 


\section{Background}

The number of people with diabetes is increasing worldwide. The World Health Organization (WHO) estimates that 285 million people had Type 2 Diabetes (T2D) in 2010 , and that this number may increase to 366 million by 2030 [1]. Diabetes is characterized by reductions in insulin production, changes in insulin resistance and glucose uptake. Over time, the increased levels of circulating glucose contribute to the development of cardiovascular and other complications, including blindness and kidney disease. The most common form of diabetes is T2D; at least $85 \%$ of all diabetes cases are T2D [2].

Physical inactivity (PA) and being overweight/obese have frequently been studied as risk factors for T2D. A number of prospective studies have investigated the effect of both PA and overweight (obesity) on incident diabetes [3-8]. Results differed. Rana et al. and Weinstein et al. showed independent associations for both physical activity and overweight/obesity with incident diabetes, with clearly a much larger impact for obesity than for physical activity [7, 8]. Results from Siegel et al. showed risks among those with obesity that were of lesser magnitude [5]. Moreover both Weinstein et al. and Siegel et al. showed only very small, maybe even negligible contributions of physical activity within those categorized as obese $[5,8]$. In turn, Manini et al. showed sitting time to be only associated with incident diabetes among obese women [4]. Kriska et al. showed that physical activity contributed to reduced risks of incident diabetes across BMI categories in a high-risk population of Pima Indians, and $\mathrm{Hu}$ et al. showed independent associations for both BMI and physical activity among those with a normal, as well as those with impaired, glucose tolerance [3, 6]. Although these studies contribute to the understanding of this subject, they were limited to a certain extent. Study populations tended to be unbalanced in that some included only one of both sexes $[4,5,7,8]$ or a high risk group $[3,6]$. In some cases, either or both BMI and physical activity were analyzed dichotomously $[5,8,9]$, instead of using a wider range of categories.

Because of the heterogeneity in methods, meta-analysis of published results from cohort studies will not result in more uniform conclusions. The InterAct-consortium was the first to publish the results of a meta-analyses beyond published results, using standardized data from a casecohort study nested within a large pan-European study $(N=27,364$ including 11,230 incident type 2 diabetes cases). Physical activity was assessed using three questions from which a four-category index was derived, and BMI was calculated using a combination of self-reported and measured values for height and weight. The conclusion was that among both men and women, higher PA levels are associated with reduced risk of developing T2D, independent of BMI [10].
In this paper we build on the standard protocols for conducting meta-analyses, which involve conducting a systematic review of the literature followed by a metaregression using published results from the included studies. Instead, in this paper, we first describe the methods used to identify existing eligible prospective cohort studies, and then how we asked researchers to re-analyze their data according to a series of predefined and harmonized proportional hazards models, so that the results could be included in our harmonized meta-regression. The aim was to examine both the independent and combined effects of PA and BMI on the development of T2D.

In contrast with the Interact study which assessed physical activity across BMI-categories, our harmonized analysis adds to understanding of the combined role of physical inactivity and high BMI to the development of T2D, using data from cohort studies of populations in and outside Europe.

\section{Methods}

\section{Identifying eligible cohorts}

A Medline search was conducted in September 2011 to identify prospective cohort studies that included data on the associations between both PA and BMI with T2D incidence. Cohort studies published between January 1989 and September 2011 were selected. The following search terms were used: Physical activity ("physical activit", 'physically active lifestyle*', 'vigorous activit", 'motor activity', 'exercise,leisure and activit"', 'recreation"', 'pedestrian", 'walking', 'running,, 'jogging, 'bicycling', 'cycling', 'skating', 'sport', 'sports', 'sporting', 'fitness', 'active', 'commuting', 'commuting activity', 'active transport', 'travel behavio? $r$ ', 'inactivity', 'sedentary behavio? $r$ ', 'television', 'tv', 'personal computer"', 'pc') and diabetes ('diabetes', 'diabetic",niddm', 'iddm'). To identify additional cohorts of interest, reference lists and reviews of the original publications were checked and we asked experts to review and supplement this list to ensure no potential cohort had been missed. This resulted in 127 cohorts that could potentially provide information about PA, BMI and T2D incidence.

Cross-sectional, case-control studies, clinical trials and other intervention studies which aimed to reduce weight and/or increase physical activity, to reduce T2D incidence were excluded. We included cohort studies according to their study characteristics rather than on the basis of the published analyses. Studies were included if: they included a generally 'healthy', predominantly white (>50\%) sample; the age range was 25-65 years at baseline; the study included measures of PA (with an indication of frequency, duration and intensity), height and weight, educational level or socio-economic status and smoking; the follow-up was at least 4 years; and incidence T2D was available at follow-up. Applying these 
criteria reduced the number of 127 potential cohorts to 35 eligible cohorts (Fig. 1).

\section{Selecting and contacting eligible cohorts}

The principal investigators (PIs) of the 35 cohorts were contacted to invite them to participate in this harmonized meta-analysis, after verifying the eligibility of their cohort. Reimbursements of costs or financial incentives were not provided. PIs were offered the possibility to delegate statistical analyses to RIVM.
Six cohorts did not respond and five cohorts declined to participate ( $N=3$ not interested, $N=2$ without reason). For 24 cohorts eligibility was double-checked in cooperation with the PIs. Three additional cohorts proved not to be eligible and thus were excluded. The detailed analysis plan was presented to 21 cohorts. Lack of resources $(N=4$ lack of resources at PIs offices, $N=3$ obligatory payments to be made by the leading authors of this paper) resulted in the exclusion of seven additional cohorts. We compared the detailed study designs

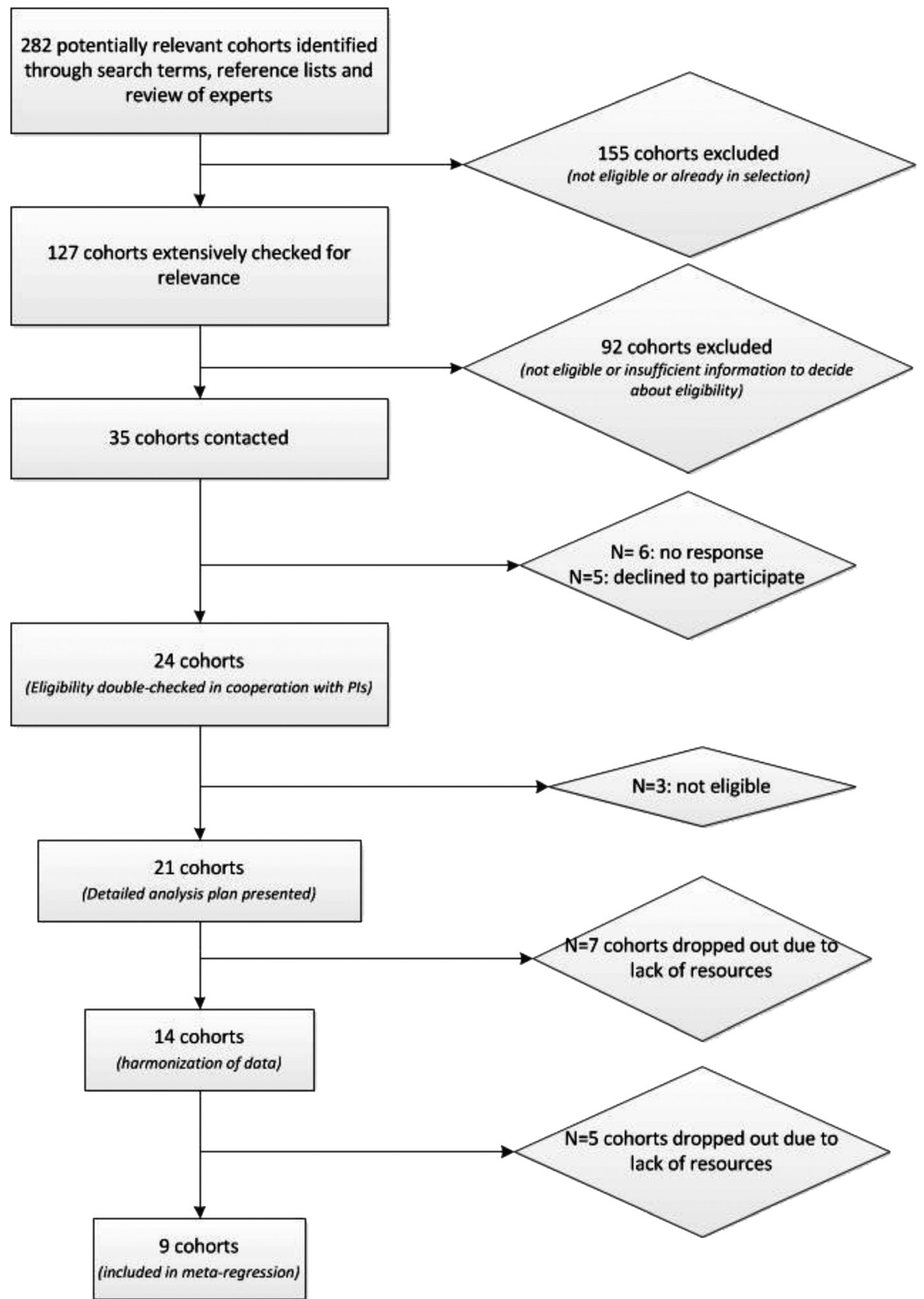

Fig. 1 Flow chart showing the selection of the cohorts 
of the 14 remaining studies and finalized an analysis plan that ensured harmonized measurement of variables and analysis for all cohorts. This whole process of contacting cohorts, double-checking eligibility and harmonizing measurements proved to be very intensive and time consuming. During this process that took approximately one and a half year, five cohorts dropped out. The meta-analysis presented in this paper included data from nine cohorts, all of which were approved by local ethical committees and were carried out in compliance with the Helsinki declaration.

\section{Data preparation}

In the analysis plan we specified the definition, measurement and cut-off values of variables. The goal was for each study to comply exactly, or as closely as possible with the following definitions of the explanatory and outcome variables.

\section{Physical activity}

Physical activity measures (frequency, intensity, and duration) for a large range of activities (see Additional file 1: Table A.1) were available. Our measures are based on two domains of activity:

- leisure time physical activities (including walking, gardening, shopping and home maintenance)

- active commuting

The measures exclude activities at work or household chores. Minutes per week spent in low, medium and high physical activity were categorized as shown in Table 1. Cut off values correspond with Global Physical Activity Guidelines [11].

\section{Body mass index (BMI)}

BMI was calculated, as weight $(\mathrm{kg})$ divided by height squared $\left(\mathrm{m}^{2}\right)$. Four BMI categories were defined, according to the WHO standard WHO [12]: underweight (BMI < $\left.18.5 \mathrm{~kg} / \mathrm{m}^{2}\right)$, normal weight $\left(18.5 \leq \mathrm{BMI}<25 \mathrm{~kg} / \mathrm{m}^{2}\right)$, overweight $\left(25 \leq \mathrm{BMI}<30 \mathrm{~kg} / \mathrm{m}^{2}\right)$ and obese $\left(\mathrm{BMI} \geq 30 \mathrm{~kg} / \mathrm{m}^{2}\right)$. Height and weight were self-reported in two studies and measured at baseline examination in the eight other studies (see Additional file 1: Table A.1).

\section{Diabetes Type 2 (T2D) incidence}

Incidence of T2D was measured within a follow-up period of (or as close as possible to) 10 years after baseline. The date of diagnosis for incident cases was

\begin{tabular}{lcc} 
Table 1 Definition of Physical activity $($ PA) categories \\
\hline Low PA & $:$ & Sum of PA-minutes $/$ week $=0$ min \\
Medium PA & $:$ & 0 min $<$ Sum of PA-minutes $/$ week $<150$ min \\
High PA & $:$ & Sum of PA-minutes $/$ week $\geq 150$ min \\
\hline
\end{tabular}

recorded as the date for onset of diabetes reported in a follow-up questionnaire or at a follow-up examination. If year was all that was available, the diagnosis date was set to July $1^{\text {st }}$ of that year. If date of diagnosis was not available, the midpoint of a time-period between examinations within which diabetes was first reported was taken.

According to clinical guidelines, a diagnosis of T2D should preferably be based on measured plasma glucose levels [13]: fasting plasma glucose $(\geq 7.0 \mathrm{mmol} / \mathrm{l})$, nonfasting plasma glucose $(\geq 11.1 \mathrm{mmol} / \mathrm{l}), 2$-h post-load plasma glucose $(\geq 11.0 \mathrm{mmol} / \mathrm{l})$ or HbA1C $(>6.5 \%)$. Alternatively, whole blood glucose levels can be used (fasting glucose: $\geq 6.1 \mathrm{mmol} / \mathrm{l}$, non-fasting glucose: $\geq 11.1 \mathrm{mmol} / \mathrm{l}$, 2-h post-load glucose: $\geq 11.1 \mathrm{mmol} / \mathrm{l})$. Three studies had measured glucose levels. The remaining six studies used self-reported questionnaire items on diabetes such as: 'Has a doctor told you, you have diabetes?' or 'Do you have type 2 diabetes?') [13]. See Additional file 1: Table A.1 for details on assessment of T2D in each study.

\section{Covariates}

Participants' age (in years), gender, educational level and smoking status were assessed at baseline. Educational level was categorized as: low (intermediate secondary school or less), medium (intermediate vocational or higher secondary education) or high (higher vocational education or university). Smoking was categorized as: non-smoker, ex-smoker or current smoker.

\section{Selection of study population in each cohort}

Only participants aged 25-65 years were included. Pregnant women, and cases with Type 1 diabetes (T1D) or T2D at baseline (prevalent cases) were excluded. Furthermore, cases with missing values on one of the following characteristics: age, gender, educational level, smoking status, physical activity, body mass index or T2D-event were excluded. For each cohort a descriptive report on the characteristics of the selected study population was compiled.

\section{Longitudinal analyses of individual cohort data}

The analysis plan included a protocol with instructions and example code in SAS for Cox-regression analyses (Table 2).

Data from six studies were analysed by their primary investigators, and in the remaining three studies the original study data were analyzed at the National Institute for Public Health and the Environment (RIVM). Analyses were done with SAS 9.2 or STATA 12. Table 2 presents an overview of the Cox-regression analyses requested for the associations between physical activity and BMI and T2D. Each model was run first without covariates and then with covariates (age, gender, educational level, smoking).

When each cohort's regression results were received at RIVM, checks were performed to verify the definition of 
Table 2 Overview of analyzed relationships (Cox regression analyses) and requested output

\begin{tabular}{|c|c|c|c|}
\hline Relation & Model 1 (no adjustment) & Model 2 (adjustment for covariates) & Requested output \\
\hline \multirow[t]{4}{*}{ Physical activity (PA) on T2D } & PA-categories: & \multirow{4}{*}{$\begin{array}{l}\text { Age, Gender, Educational level, } \\
\text { Smoking, BMI-categories }\end{array}$} & \multirow{4}{*}{$\begin{array}{l}\text { HRs, Betas, SEs and } 95 \% \\
\text { Confidence intervals }\end{array}$} \\
\hline & Low PA & & \\
\hline & Medium PA & & \\
\hline & High $\mathrm{PA}^{\mathrm{a}}$ & & \\
\hline \multirow[t]{5}{*}{ Body Mass Index (BMI) on T2D } & BMl-categories: & \multirow{5}{*}{$\begin{array}{l}\text { Age, Gender, Educational level, } \\
\text { Smoking, PA-categories }\end{array}$} & \multirow{5}{*}{$\begin{array}{l}\text { HRs, Betas, SEs and } 95 \% \\
\text { Confidence intervals }\end{array}$} \\
\hline & Underweight & & \\
\hline & Normal weight ${ }^{a}$ & & \\
\hline & Overweight & & \\
\hline & Obesity & & \\
\hline \multirow{13}{*}{$\begin{array}{l}\text { Combined effect of Physical } \\
\text { activity (PA) and Body Mass } \\
\text { Index (BMI) on T2D }\end{array}$} & Combined categories: & \multirow{13}{*}{$\begin{array}{l}\text { Age, Gender, Educational level, } \\
\text { Smoking }\end{array}$} & \multirow{13}{*}{$\begin{array}{l}\text { HRs, Betas, SEs and } 95 \% \\
\text { Confidence intervals }\end{array}$} \\
\hline & High PA - Normal weight ${ }^{a}$ & & \\
\hline & Low PA - Underweight & & \\
\hline & Low PA - Normal weight & & \\
\hline & Low PA - Overweight & & \\
\hline & Low PA - Obesity & & \\
\hline & Medium PA - Underweight & & \\
\hline & Medium PA - Normal weight & & \\
\hline & Medium PA - Overweight & & \\
\hline & Medium PA - Obesity & & \\
\hline & High PA - Underweight & & \\
\hline & High PA - Overweight & & \\
\hline & High PA - Obesity & & \\
\hline
\end{tabular}

${ }^{\mathrm{a}}$ Reference category

variables and that analyses had been conducted according to the protocol. This entailed extensive communication between the PIs of each study and the first author of this paper. Results were returned on average 4 times before they were fully compliant with the protocol.

In the subset of studies for which original data were analyzed at RIVM, the proportional hazard assumption for the explanatory variables in Cox regression model was tested [14].

\section{Meta survival regression}

Because of differences within the 9 study populations, a random effects model was used [15]. Heterogeneity was tested with a likelihood ratio test. Hazard ratios and standard errors were pooled using the restricted maximum likelihood method.

Summary estimates for categories of physical activity with reference level high physical activity and categories of BMI with reference level normal weight, were calculated. These models were unadjusted or adjusted for age, gender, educational level, and smoking. Physical activity models were additionally adjusted for BMI and BMI models were additionally adjusted for physical activity.

\section{Results}

Descriptive statistics of the nine included studies are presented in Table 3. $N=117,878$ participants were included; individual studies varied in size from 1087 (KIHD) to 86,368 (CPS-II), the weight of the latter was substantial in the pooled analysis, being much larger than other studies. Three studies consisted entirely of male participants (BHRS [16], CaPS [17], KIHD) and one study consisted entirely of female participants (ALSWH [18]). Mean follow-up time was 9.1 years. A total of 11,237 incident T2D cases were recorded. The mean time to T2D event ranged from 4.2 years (KIHD) to 8.8 years (PALS [19]).

The assumption of proportional hazards was not met for the lowest category of BMI, underweight $(P<0.001)$. Since numbers in this category were small and because of the non-proportionality of hazards, underweight was not included in the results.

The results of the meta-analysis showing the relationships between physical activity and $\mathrm{T} 2 \mathrm{D}$ are shown in Table 4 (also see Additional file 1: Figure B1 and B2). In the unadjusted model, the risk of T2D was $64 \%$ higher in the low PA category compared with the high PA category. Adjustment for confounders attenuated this estimate to 
Table 3 Descriptive statistics of 9 individual cohort studies included in the meta-analysis, $n=119,396$ participants

\begin{tabular}{|c|c|c|c|c|c|c|c|c|c|c|}
\hline & Cohort & Country & $N$ & Mean age & $\%$ male & $\begin{array}{l}\text { Mean time to } \\
\text { T2D event (Years) }\end{array}$ & $\begin{array}{l}\text { \% Incident cases T2D } \\
\text { (Absolute number) }\end{array}$ & $\begin{array}{l}\text { PA categories \% (Absolute } \\
\text { number) }\end{array}$ & BMI categories \% (Absolute number) & $\begin{array}{l}\text { Mean } \\
\text { BMl }\end{array}$ \\
\hline \multirow[t]{4}{*}{1} & \multirow{4}{*}{$\begin{array}{l}\text { Australian Longitudinal Study on } \\
\text { Women's Health (ALSWH) [18] }\end{array}$} & \multirow[t]{4}{*}{ Australia } & \multirow[t]{4}{*}{9599} & \multirow[t]{4}{*}{49.6} & \multirow[t]{4}{*}{$0 \%$} & \multirow[t]{4}{*}{4.9} & \multirow[t]{4}{*}{$4.6 \%(441)$} & Low $=17.7 \%(1695)$ & underweight $=1.6 \%(140)$ & \multirow[t]{4}{*}{26.2} \\
\hline & & & & & & & & Med = $25.8 \%(2474)$ & normal weight = $47.8 \%$ (4592) & \\
\hline & & & & & & & & \multirow[t]{2}{*}{ High = $56.6 \%(5430)$} & mod overweight = $31.6 \%$ (3036) & \\
\hline & & & & & & & & & obese = $19.1 \%(1831)$ & \\
\hline \multirow[t]{4}{*}{2} & \multirow{4}{*}{$\begin{array}{l}\text { The Australian Diabetes, Obesity } \\
\text { and Lifestyle study (AusDiab) [24] }\end{array}$} & \multirow[t]{4}{*}{ Australia } & \multirow[t]{4}{*}{4327} & \multirow[t]{4}{*}{47.1} & \multirow[t]{4}{*}{$46 \%$} & \multirow[t]{4}{*}{4.3} & \multirow[t]{4}{*}{$3.0 \%(130)$} & Low $=39.6 \%(1712)$ & underweight = 0.8 \% (35) & \multirow[t]{4}{*}{26.5} \\
\hline & & & & & & & & Med $=57.8 \%(2503)$ & normal weight = $40.4 \%$ (1748) & \\
\hline & & & & & & & & \multirow[t]{2}{*}{ High = $2.6 \%(112)$} & mod overweight = $39.5 \%$ (1709) & \\
\hline & & & & & & & & & obese = $19.3 \%(835)$ & \\
\hline \multirow[t]{4}{*}{3} & \multirow{4}{*}{$\begin{array}{l}\text { British Regional Heart Study } \\
\text { (BRHS) [16] }\end{array}$} & \multirow{4}{*}{$\begin{array}{l}\text { United } \\
\text { Kingdom }\end{array}$} & \multirow[t]{4}{*}{4720} & \multirow[t]{4}{*}{49.3} & \multirow[t]{4}{*}{$100 \%$} & \multirow[t]{4}{*}{7.0} & $1.7 \%(82)$ & Low $=31.3 \%(2547)$ & underweight = $0.4 \%$ (19) & 25.4 \\
\hline & & & & & & & & Med $=14.8 \%(698)$ & normal weight = 46.4 \% (2188) & \\
\hline & & & & & & & & High $=54.0 \%(2547)$ & mod overweight = $46.3 \%$ (2184) & \\
\hline & & & & & & & & & obese = $7.0 \%$ (329) & \\
\hline 4 & Caerphilly Prospective Study & United & 2034 & 56.6 & $100 \%$ & 4.7 & $4.9 \%(99)$ & Low $=0 \%(0)$ & underweight = $1.2 \%(25)$ & 26.4 \\
\hline & & & & & & & & Med $=18.6 \%(378)$ & normal weight = $32.5 \%$ (660) & \\
\hline & & & & & & & & High = $81.4 \%(1656)$ & mod overweight = $52.4 \%$ (1065) & \\
\hline & & & & & & & & & obese = $14.0 \%(284)$ & \\
\hline 5 & Cancer prevention study-\|I & United & 86368 & 58.7 & $42 \%$ & 6.3 & $11.5 \%$ (9948) & Low $=1.3 \%(1119)$ & underweight = $1.1 \%$ (969) & 26.1 \\
\hline & & & & & & & & Med = $30.3 \%$ (26166) & normal weight $=43.8 \%$ (37851) & \\
\hline & & & & & & & & High = $68.4 \%$ (59083) & mod overweight $=39.5 \%$ (34123) & \\
\hline & & & & & & & & & obese $=15.5 \%$ (13425) & \\
\hline 6 & Doetinchem Cohort Study [25] & & 3401 & 46.7 & $44 \%$ & 5.6 & $2.0 \%(67)$ & Low $=0.7 \%(24)$ & underweight = $0.8 \%(27)$ & 25.6 \\
\hline & & Netherlands & & & & & & Med $=2.1 \%(71)$ & normal weight $=47.2 \%$ (1605) & \\
\hline & & & & & & & & High $=97 \%(3306)$ & mod overweight = $40.7 \%$ (1383) & \\
\hline & & & & & & & & & obese = $11.4 \%$ (386) & \\
\hline 7 & Kuopio Ischaemic Heart Disease & Finland & 1087 & 51.7 & $100 \%$ & 4.2 & $5.1 \%(55)$ & Low $=0.6 \%(6)$ & underweight = $0.1 \%(1)$ & 26.8 \\
\hline & & & & & & & & Med $=11.4 \%(124)$ & normal weight $=34.4 \%(374)$ & \\
\hline & & & & & & & & High $=88 \%(957)$ & mod overweight $=50.4 \%$ (548) & \\
\hline & & & & & & & & & obese = $15.1 \%(164)$ & \\
\hline 8 & Physical Activity Longitudinal & Canada & 1487 & 41.0 & $48 \%$ & 8.8 & $2.2 \%(32)$ & Low $=2.1 \%(31)$ & underweight = $2.2 \%(32)$ & 25.2 \\
\hline & Study (PALS) [19] & & & & & & & Med $=30 \%(446)$ & normal weight $=50.7 \%(754)$ & \\
\hline & & & & & & & & High = $67.9 \%(1010)$ & mod overweight $=36.3 \%$ (540) & \\
\hline & & & & & & & & & obese = $10.8 \%(161)$ & \\
\hline
\end{tabular}


Table 3 Descriptive statistics of 9 individual cohort studies included in the meta-analysis, $n=119,396$ participants (Continued)

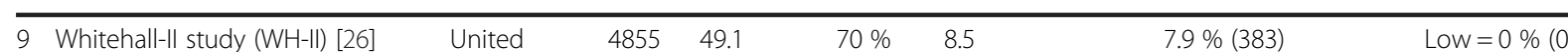

Med $=10 \%(508)$

underweight $=0.95(46)$

25.3

Kingdom

High $=89.5 \%(4347)$

normal weight $=51.99(2524)$

mod overweight $=38.00(1845)$

obese $=9.06(440)$ 
Table 4 Meta- analysis of baseline physical activity categories as determinant of T2D development

\begin{tabular}{|c|c|c|c|c|c|c|}
\hline \multirow[t]{2}{*}{ Physical activity on T2D } & \multirow[b]{2}{*}{$N$} & \multicolumn{2}{|c|}{ Model $1^{a}$} & \multicolumn{3}{|c|}{ Model $2^{b}$} \\
\hline & & $\mathrm{HR}$ & $95 \% \mathrm{Cl}$ & $\mathrm{HR}$ & $95 \% \mathrm{Cl}$ & Heterogeneity $p$-value \\
\hline High physical activity & 9 & 1.00 & & 1.00 & & \\
\hline Medium physical activity & 9 & 1.11 & $0.86-1.43$ & 1.08 & $1.04-1.13$ & $1^{d}$ \\
\hline Low physical activity & $7^{c}$ & 1.64 & $1.45-1.85$ & 1.23 & $1.09-1.39$ & $1^{d}$ \\
\hline
\end{tabular}

${ }^{a}$ Model 1: T2D = physical activity level categories

${ }^{\mathrm{b}}$ Model 2: Model 1 + age + gender + educational level + smoking + body mass index categories

'No estimates for CaPS and WH-II

${ }^{d}$ Between study variance is estimated as zero

1.23 (95 \% CI: 1.09-1.39). For medium PA risk estimates were also attenuated, but to a lesser degree.

Relationships between baseline BMI and risk of developing T2D are shown in Table 5 (also see Additional file 1: Figure B3 and B4). The associations between being overweight or obese and risk of T2D were stronger than the associations of lower levels of physical activity and T2D. The summary hazard ratios for developing T2D in participants who were overweight or obese were 2.33 (95\% CI: 1.95-2.78) and 6.10 (95 \% CI: 4.63-8.04) respectively.

The combined effect of physical activity and body mass index levels on T2D was assessed by creating separate indicators for combinations of PA and BMI. Results are in Table 6 and graphically shown in Fig. 2.

The single reference group in this analysis was thigh physical activity/normal weight'. The highest hazard ratio was among individuals who were both obese and had low physical activity (HR 7.43, 95 \% CI 3.47-15.89). Except for 'medium physical activity/normal weight', hazard ratios were higher for all other combinations of PA and BMI than in the comparison group 'high physical activity/normal weight' (significance level $p<0.05$ ). Within the overweight and obese BMI categories, the summary hazard ratios of T2D decreased with higher levels of physical activity. The $\mathrm{p}$ value for the test of linear trend across the three overweight combinations (low PA/overweight, medium PA/ overweight, high PA/overweight) in the adjusted model was $p=0.0076$ and across the three obese combinations (low PA/obese, medium PA/obese, high PA/obese) in the adjusted model $: p=0.5780$.

\section{Discussion}

In contrast with previous studies which have assessed physical activity across BMI-categories, our harmonized analysis adds to understanding of the combined role of physical inactivity and high BMI in the development of T2D, using data from cohort studies of population samples from within and outside Europe. In this metaanalysis of harmonized data from 9 prospective cohort studies - in mutually adjusted models - being overweight, obese (compared to normal weight) and having low physical activity (compared to high physical activity) were associated with an increased risk of incident type 2 diabetes (hazard ratios 2.33, 95 \% CI 1.95-2.78; 6.10, 95 \% CI: 4.63-8.04, and 1.23, 95 \% CI: 1.09-1.39, respectively). Individuals who were both obese and had low physical activity had a 7.4-fold (95 \% CI 3.47-15.89) increased risk of type 2 diabetes, compared with normal weight, high physically active participants.

This study showed slightly weaker individual associations between physical activity, overweight and obesity and diabetes than previous studies have shown. For example, Jeon et al. calculated a summary relative risk based on 10 prospective cohort studies of 1.45 (95 \% CI: $1.20-1.72$ ) for being sedentary compared to regular participation in moderate physical activity [20]. Aune et al. calculated summary relative risks for several physical activity measures based on a mix of prospective cohort, case-cohort, nested case-control studies and randomized trials. In their analysis for total physical activity, 14 prospective cohort studies were included. The summary estimate for low versus high total physical activity was 1.54 (95 \% CI: 1.41-1.69). For leisure time physical activity they calculated a summary estimate of 1.35 (95 \% CI: 1.27-1.41) based on 55 prospective cohort studies [21]. The stronger association in the Jeon et al. study may be explained by the fact that they included a large range of physical activity domains. In contrast, our PA-measure

Table 5 Meta-analysis of baseline body mass index categories as determinants of T2D development

\begin{tabular}{|c|c|c|c|c|c|c|}
\hline \multirow[t]{2}{*}{ Body mass index on $\mathrm{T} 2 \mathrm{D}$} & \multirow[b]{2}{*}{ N } & \multicolumn{2}{|c|}{ Model $1^{a}$} & \multicolumn{3}{|c|}{ Model $2^{b}$} \\
\hline & & $\overline{H R}$ & $95 \% \mathrm{Cl}$ & $\overline{H R}$ & $95 \% \mathrm{Cl}$ & Heterogeneity $p$-value \\
\hline Normal weight & 9 & 1.00 & & 1.00 & & \\
\hline Overweight & 9 & 2.46 & $2.07-2.91$ & 2.33 & $1.95-2.78$ & 0.096 \\
\hline Obesity & 9 & 6.68 & $4.94-9.03$ & 6.10 & $4.63-8.04$ & $<.0001$ \\
\hline
\end{tabular}

${ }^{\mathrm{a}}$ Model 1: $\mathrm{T} 2 \mathrm{D}=\mathrm{BMI}$ categories

${ }^{\mathrm{b}}$ Model 2: Model $1+$ age + gender + educational level + smoking + physical activity level categories 
Table 6 Meta-analysis of combined classes of baseline physical activity (PA) and baseline body mass index on development of T2D

\begin{tabular}{|c|c|c|c|}
\hline \multirow[t]{2}{*}{ Combined PA x BMI classes } & \multirow[b]{2}{*}{$N$} & \multirow{2}{*}{$\begin{array}{l}\text { Model } 1^{\text {a }} \\
\text { HR }(95 \% \text { Cl) }\end{array}$} & \multirow{2}{*}{$\begin{array}{l}\text { Model } 2^{\mathrm{b}} \\
\text { HR }(95 \% \text { Cl) }\end{array}$} \\
\hline & & & \\
\hline High PA, normal weight & & 1.00 & 1.00 \\
\hline Medium PA, normal weight & 9 & $0.80(0.46-1.38)$ & $0.84(0.50-1.39)$ \\
\hline Low PA, normal weight & 6 & $1.75(1.19-2.58)$ & $1.61(1.09-2.37)$ \\
\hline High PA, overweight & 9 & $2.38(1.83-3.10)$ & $2.26(1.74-2.93)$ \\
\hline Medium PA, overweight & 9 & $2.52(1.94-3.28)$ & $2.45(1.87-3.20)$ \\
\hline Low PA, overweight & 7 & $3.00(2.31-3.88)$ & $2.86(1.93-4.22)$ \\
\hline High PA, obese & 9 & $6.62(4.39-10.00)$ & $6.13(4.25-8.84)$ \\
\hline Medium PA, obese & 9 & $7.21(4.16-12.51)$ & $6.93(4.20-11.43)$ \\
\hline Low PA, obese & 7 & $8.07(3.91-16.67)$ & $7.43(3.47-15.89)$ \\
\hline
\end{tabular}

${ }^{a}$ Model 1: T2D = combined physical activity level and $\mathrm{BMI}$ categories

${ }^{\mathrm{b}}$ Model 2: Model $1+$ age + gender + educational level + smoking

was based on only two domains of physical activity: leisure time physical activity and transport-related physical activity (specifically active commuting). Aune et al. showed a weaker association based on leisure time physical activity alone than for total physical activity. Our summary estimate for low versus high physical activity was 1.23 (95 \% CI: 1.09-1.39). Abdullah et al. calculated a summary relative risk based on 18 prospective cohort studies of 7.28 (95 \% CI: 6.47-8.28) for obesity and 2.92 (95 \% CI: 2.57-3.32) for overweight, compared with normal weight [22]. Our summary estimates were 6.10 (95\% CI: 4.63-8.04) and 2.33 (95\% CI: 1.95-2.78) respectively.

Furthermore we observed stronger associations with T2D for obesity than for physical activity. This is in line with previous studies. In a systematic review, Fogelholm found that in six out of eight included studies the combination of being obese and physically active was a greater health hazard in terms of T2D than having normal weight and being inactive [23]. The InterActconsortium concluded that for both men and women lower levels of physical activity were associated with an increased risk of T2D across BMI-categories [10]. Similarly, our findings showed decreasing HRs for T2D incidence within the overweight and obese BMI categories for increasing levels of physical activity. This trend was statistically significant among those categorized as overweight ( $\mathrm{p}$ for trend: 0.0076 ) but not for those categorized as obese ( $\mathrm{p}$ for trend: 0.5780). In future studies, using objective measures for physical activity, as well as height and weight, could help to further assess the relative importance of adiposity and physical activity in relation to T2D.

The strength of our study is that results are not based on published results, but on (re) analysis of data from individual cohorts, following a standardized protocol. This harmonized analysis reduces the heterogeneity of the study results. As the studies were not published, we could not assess publication bias in the standard way. However, there was potential for bias because data from only nine of the 21 eligible prospective cohort studies could be included. As the cohorts that were not included have not published on the combined effect of physical activity and BMI on T2D, it is difficult to judge the direction or the extent of bias in our summary estimates. Despite our efforts to standardize, not all differences between cohorts could be resolved, because the PA questionnaires and methods of T2D case ascertainment differed across cohorts. The quality of measurement of

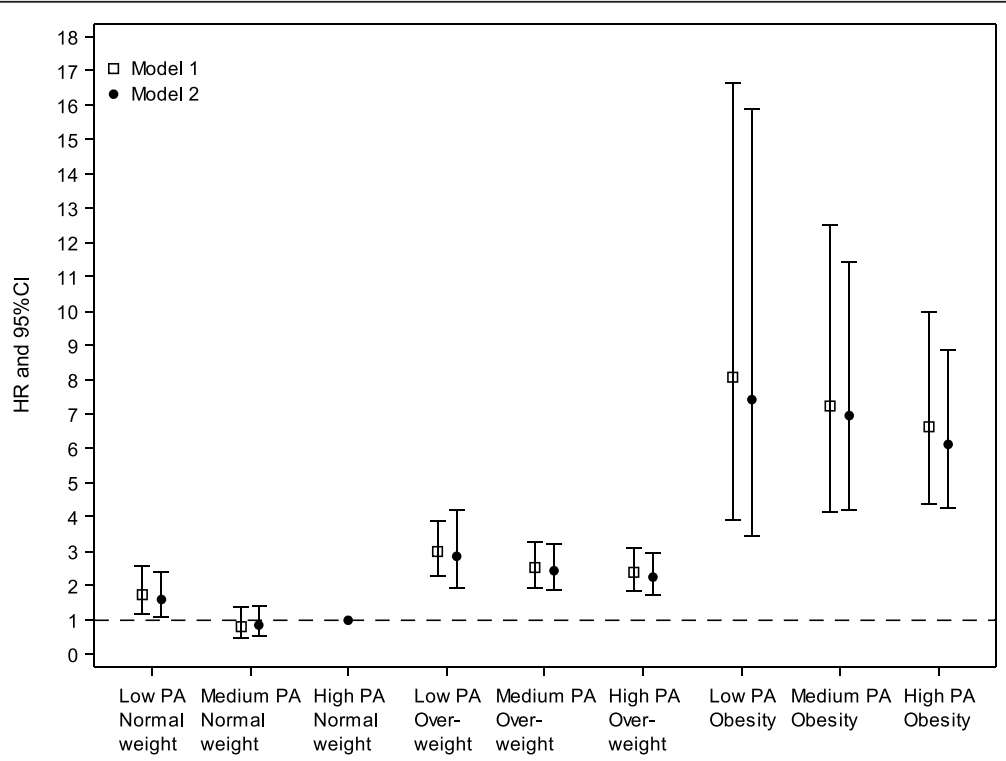

Fig. 2 Meta-analysis of combined classes of baseline physical activity and body mass index on T2D development. Model 1: T2D =combined physical activity level and BMI categories. Model 2: Model $1+$ age + gender + educational level + smoking 
overweight and obesity was likely to have been higher than for PA, which was measured using quite crude methods in some studies. This would have affected the effect sizes, and may have consequences for interpreting the relative importance of both risk factors in T2D development. Use of objective measures of PA in future studies will improve these limitations.

\section{Conclusions}

Our study demonstrates important associations between increasing levels of BMI and decreasing levels of physical activity on the development of T2D. Participants who were obese and who had the lowest levels of physical activity were most at risk for developing T2D. Our findings underline the importance of maintaining a healthy weight and also of being physically active in order to reduce risk of developing T2D.

\section{Additional file}

Additional file 1: Table A.1 - Characteristics of cohort studies included in the meta-analysis. Figure B.1. Hazard ratios and $95 \% \mathrm{Cl}$ of baseline low physical activity on development of T2D. Model is adjusted for age, gender, educational level, smoking and BMI. Figure B.2. Hazard ratios and $95 \% \mathrm{Cl}$ of baseline medium physical activity on development of T2D. Model is adjusted for age, gender, educational level, smoking and BMI. Figure B.3. Hazard ratios and $95 \% \mathrm{Cl}$ of baseline overweight on development of T2D. Model is adjusted for age, gender, educational level, smoking and physical activity. Figure B.4. Hazard ratios and $95 \% \mathrm{Cl}$ of baseline obesity on development of T2D. Model is adjusted for age, gender, educational level, smoking and physical activity. (DOCX 39 kb)

\section{Competing interests}

The authors declare that they have no competing interests.

\section{Authors' contributions}

Study concept and design: LC, WWV, WB. Acquisition and verification of data: LC. Statistical analysis: LC, BH, CLC, MK, CN, BJ, KR, GD. Drafting the article: LC, WWV, GD. Critical revision: All authors. All authors read and approved the final manuscript.

\section{Acknowledgements}

We are grateful to all those who contributed to data collection at the study sites. We would like to thank Hendriek Boshuizen for statistical support and Annemieke Spijkerman for her help in structuring basic concepts of this meta-analysis. The Whitehall II study and M. Kivimäki are supported by the UK Medical Research Council (K013351), the British Heart Foundation, the US National Institutes of Health, and the UK Economic and Social Research Council. The Australian Longitudinal Study on Women's Health is funded by the Australian Government Department of Health. The American Cancer Society funds the creation, maintenance, and updating of the Cancer Prevention Study II cohort . B.J. Jefferis was supported by an NIHR Post-Doctoral Fellowship (2010-03-023). The British Regional Heart Study is supported by a British Heart Foundation programme grant (RG/13/16/30528). The National Institute of Health Research and British Heart Foundation had no role in the design and conduct of the study; collection, management, analysis, and interpretation of the data; preparation, review, or approval of the manuscript; and decision to submit the manuscript for publication. The views expressed in this publication are those of the author(s) and not necessarily those of the National Institute for Health Research or the British Heart Foundation. Collaborators per study site are: Wendy Brown for the Australian Longitudinal Study on Women's Health (ALSWH), Yoav Ben-Shlomo for the : Caerphilly Prospective Study (CaPS), Christina Newton for the Cancer prevention study-ll Nutrition Cohort (CPS-II), Kimmo Ronkainen, Jari Antero Laukkanen and Jussi
Kauhanen for the Kuopio Ischaemic Heart Disease Risk Factor Study (KIHD), Cora Lynn Craig for the Physical Activity Longitudinal Study (PALS), Mika Kivimäki and Adam Tabak for the Whitehall-II study (WH-II), Susan Picavet for the Doetinchem Cohort Study, Bethany Howard for The Australian Diabetes, Obesity and Lifestyle study (AusDiab) and Barbara Jefferis for the British Regional Heart Study (BRHS)

\section{Author details}

${ }^{1}$ National Institute for Public Health and the Environment, Bilthoven, The Netherlands. ${ }^{2}$ Baker IDI Heart and Diabetes Institute, Melbourne, VIC, Australia. ${ }^{3}$ Canadian Fitness and Lifestyle Research Institute, Ottawa, Canada. ${ }^{4}$ Department of Epidemiology and Public Health, University College London, London, UK. ${ }^{5}$ Department of Primary Care and Population Health, University College London, London, United Kingdom. ${ }^{6}$ Institute of Public Health and Clinical Nutrition, University of Eastern Finland, Kuopio, Finland. ${ }^{7}$ School of Human Movement and Nutrition Sciences, University of Queensland, Brisbane, Australia. ${ }^{8}$ Department of Clinical Epidemiology, School of Social and Community Medicine, University of Bristol, Bristol, UK. ${ }^{9}$ Department of Internal Medicine, Lapland Central Hospital, Rovaniemi, Finland.

Received: 23 July 2015 Accepted: 4 November 2015

Published online: 01 December 2015

\section{References}

1. Wild S, Roglic G, Green A, Sicree R, King H. Global prevalence of diabetes: estimates for the year 2000 and projections for 2030. Diabetes Care. 2004;27(5):1047-53.

2. International Diabetes Federation. IDF Diabetes Atlas. 6th ed. Brussels: International Diabetes Federation; 2013.

3. Kriska AM, Saremi A, Hanson RL, Bennett PH, Kobes S, Williams DE, et al. Physical activity, obesity, and the incidence of type 2 diabetes in a high-risk population. Am J Epidemiol. 2003;158(7):669-75.

4. Manini TM, Lamonte MJ, Seguin RA, Manson JE, Hingle M, Garcia L, et al. Modifying effect of obesity on the association between sitting and incident diabetes in post-menopausal women. Obesity (Silver Spring). 2014;22(4):1133-41.

5. Siegel LC, Sesso HD, Bowman TS, Lee IM, Manson JE, Gaziano JM. Physical activity, body mass index, and diabetes risk in men: a prospective study. Am J Med. 2009;122(12):1115-21.

6. Hu G, Lindstrom J, Valle TT, Eriksson JG, Jousilahti P, Silventoinen $\mathrm{K}$, et al. Physical activity, body mass index, and risk of type 2 diabetes in patients with normal or impaired glucose regulation. Arch Intern Med. 2004;164(8):892-6.

7. Rana JS, Li TY, Manson JE, Hu FB. Adiposity compared with physical inactivity and risk of type 2 diabetes in women. Diabetes Care. 2007;30(1):53-8.

8. Weinstein AR, Sesso HD, Lee IM, Cook NR, Manson JE, Buring JE, et al. Relationship of physical activity vs body mass index with type 2 diabetes in women. JAMA. 2004;292(10):1 188-94.

9. Hu G, Qiao Q, Tuomilehto J, Balkau B, Borch-Johnsen K, Pyorala K. Prevalence of the metabolic syndrome and its relation to all-cause and cardiovascular mortality in nondiabetic European men and women. Arch Intern Med. 2004;164(10):1066-76.

10. The InterAct Consortium. Physical activity reduces the risk of incident type 2 diabetes in general and in abdominally lean and obese men and women: the EPIC-InterAct Study. Diabetologia. 2012;55(7):1944-52.

11. Global Recommendations on Physical Activity for Health. WHO Guidelines Approved by the Guidelines Review Committee. Geneva: World Health Organization; 2010.

12. WHO. Obesity: preventing and managing the global epidemic. Report of a WHO Consultation. Geneva: World Health Organization; 2000.

13. American Association of Clinical Endocrinologists. American Association of Clinical Endocrinologists/American College of Endocrinology statement on the use of hemoglobin A1c for the diagnosis of diabetes. Endocr Pract. 2010;16(2):155-6.

14. Lin DYWL, Ying Z. Checking the Cox model with cumulative sums of martingale-based residuals. Biometrika. 1993;80:573-81.

15. Stijnen T, Hamza TH, Özdemir P. Random effects meta-analysis of event outcome in the framework of the generalized linear mixed model with applications in sparse data. Stat Med. 2010;29(29):3046-67. 
16. Walker M, Whincup PH, Shaper AG. The British Regional Heart Study 1975-2004. Int J Epidemiology. 2004;33(6):1185-92.

17. The Caerphilly and Speedwell Collaborative Group. Caerphilly and Speedwell collaborative heart disease studies. J Epidemiol Community Health. 1984;38:259-62

18. Brown WJ, Bryson L, Byles JE, Dobson AJ, Lee C, Mishra G, et al. Women's Health Australia: recruitment for a national longitudinal cohort study. Women \& health. 1999:28(1):23-40.

19. Craig CL, Gauvin L, Cragg S, Katzmarzyk PT, Stephens T, Russell SJ, et al. Towards a social epidemiological perspective on physical activity and health: the aims, design, and methods of the Physical Activity Longitudinal Study (PALS). J Phys Act Health. 2005;2:272-84

20. Jeon CY, Lokken RP, Hu FB, van Dam RM. Physical activity of moderate intensity and risk of type 2 diabetes: a systematic review. Diabetes Care. 2007:30(3):744-52

21. Aune D, Norat T, Leitzmann M, Tonstad S, Vatten LJ. Physical activity and the risk of type 2 diabetes: a systematic review and dose-response metaanalysis. Eur J Epidemiol. 2015;30(7):529-42.

22. Abdullah A, Peeters A, de Courten M, Stoelwinder J. The magnitude of association between overweight and obesity and the risk of diabetes: a meta-analysis of prospective cohort studies. Diabetes Res Clin Pract. 2010;89(3):309-19.

23. Fogelholm M. Physical activity, fitness and fatness: relations to mortality, morbidity and disease risk factors. A systematic review. Obes Rev. 2010;11(3):202-21

24. Dunstan DW, Zimmet PZ, Welborn TA, Cameron AJ, Shaw J, de Courten M et al. The Australian Diabetes, Obesity and Lifestyle Study (AusDiab) — methods and response rates. Diabetes Res Clin Pract. 2002:57(2):119-29.

25. Verschuren WM, Blokstra A, Picavet HS, Smit HA. Cohort profile: the Doetinchem cohort study. Int J Epidemiol. 2008;37(6):1236-41.

26. Marmot M, Brunner E. Cohort Profile: the Whitehall II study. Int J Epidemiol. 2005:34(2):251-6

\section{Submit your next manuscript to BioMed Central and take full advantage of:}

- Convenient online submission

- Thorough peer review

- No space constraints or color figure charges

- Immediate publication on acceptance

- Inclusion in PubMed, CAS, Scopus and Google Scholar

- Research which is freely available for redistribution 